\title{
Effects of chronic flexed wrist posture on the elasticity and cross- sectional area of the median nerve at the carpal tunnel among chronic stroke patients
}

\author{
Hulya Aslan', Pinar Doruk Analan²
}

${ }^{1}$ Department of Radiology, ${ }^{2}$ Department of Physical Medicine and Rehabilitation, Baskent University Faculty of Medicine, Adana, Turkey.

\begin{abstract}
Aim: To investigate the effects of chronic flexed wrist posture following spasticity on the elasticity and cross-sectional area (CSA) of the median nerve at the carpal tunnel among chronic stroke patients. Material and method: This prospective study included 24 consecutive patients (mean age, $56.5 \pm 11.56$ years) with unilateral wrist spasticity following a stroke in a chronic phase. The CSA of the median nerve was measured by ultrasound (US). The elasticity was measured by Virtual Touch tissue imaging quantification (VTIQ; Siemens, Erlangen, Germany). SWV and CSA of the median nerves of the affected and unaffected sides for each patient were compared. The correlations between duration of time since the stroke, SWV and CSA of the median nerve were assessed. The interobserver agreement was assessed. Results: The CSA of the median nerve at the affected side was significantly lower than that of the unaffected side $(\mathrm{p}=0.03)$. The SWV of the median nerve at the affected side was significantly higher than that on the unaffected side $(p<0.001)$. The interobserver agreement was excellent for both CSA and SWV measurements. There was a negatively fair correlation between CSA at the affected side and duration of time since stroke $(r=-0.58, p<0.05)$. The SWV of the median nerve at the affected side was not correlated with the duration of time since stroke $(r \leq 0.3, p \geq 0.05)$. Conclusions: These results suggest that chronic flexed wrist posture may cause atrophy of the median nerve due to chronic compression after stroke and increase in the stiffness of the median nerve.
\end{abstract}

Keywords: shear wave elastography; stroke; wrist spasticity; median nerve; posture

\section{Introduction}

Spasticity is a velocity-dependent increase in the muscle tone as a part of the upper motor neuron syndrome. Post stroke spasticity is characterized by hyperexcitability, a rapid rise in excitation and stereotypical postures. In the upper extremity impairment the hemiplegic posture consists of shoulder adduction, forearm pronation, and elbow, wrist, and finger flexion [1,2]. Compression neuropathy occurs when a peripheral nerve is chronically

Received 16.08.2017 Accepted 14.10.2017

Med Ultrason

2018, Vol. 20, No 1, 71-75

Corresponding author: Hulya Aslan, MD

Department of Radiology,

Baskent University Faculty of Medicine,

Adana Dr. Turgut Noyan Teaching and

Medical Research Center, 01250, Adana, Turkey

Phone: +90-322-327 2727, Fax: +90-322-327 1274

E-mail: hul_yaaslan@hotmail.com exposed to increased pressure, often within an anatomic compartment. The carpal tunnel is an anatomic compartment delimited by the transverse carpal ligament and median nerve runs through the carpal tunnel. Post stroke flexed posture of the wrist may affect the pressure of the carpal tunnel and compress the median nerve.

Median nerve ultrasound (US) is a non-invasive, inexpensive, and useful adjunctive tool that allows the median nerve to be visualized at real time. Also, the improvements in US technology have made it possible to measure tissue stiffness noninvasively by elastography. A recently published study showed that posture-induced changes could be detected by Shear-Wave Elastography (SWE) at the median nerve [3]. Previous US studies have reported conflicting results on whether median nerve cross-sectional area (CSA) is altered in different wrist postures [4-6]. Although the effect of acute postural changes to the median nerve has been studied, the chronic effects of postural changes to the median nerve 
have not been clearly identified yet. Therefore, the aim of this study was to analyze the effects of chronic flexed posture on the elasticity and CSA of the median nerve among stroke patients.

\section{Material and methods}

\section{Subjects}

This study was approved by the Institutional Review Board and Ethics Committee and supported by the Institutional Research Fund. Written informed consent was obtained from all patients. All the patients were asymptomatic for median and brachial neuropathies. None of the patients had diabetes, hypothyroidism, RA, or Down syndrome.

The inclusion criteria were: unilateral wrist spasticity, at least Modified Ashworth Scale (MAS) 1 on the hemiplegic side; age above 18 years; more than 3 weeks from the onset of stroke symptoms and having flexed wrist posture; sufficient cognitive and communication ability to be able to give written informed consent. The exclusion criteria were as follows: established contracture or other neurological impairments without stroke; received botulinum toxin or treatments involving neurolytic agent (such as phenol or alcohol) injections within the past 6 months; prior surgery or major trauma to the upper extremity and any other muscular disorders that may have affected the wrist; previous diagnosis of carpal tunnel syndrome (CTS) and clinical evidence of CTS.

Power analysis during the biostatistical preliminary assessment indicated a study population of 24 patients with $95 \%$ confidence level and $80 \%$ power, so, a total of 29 patients were enrolled in the study. Of these, two patients were excluded for having bilateral wrist spasticity and three for having taken botulinum toxin injections 3 months ago. Thus, 24 patients were finally included in the study.

\section{US and Shear Wave Elastography (SWE) examinations}

The US and SWE examinations were performed with a US system (Acuson S 2000; Siemens, Erlangen, Germany). After the clinical evaluation, patients underwent SWE of the median nerves on both paretic and nonparetic sides. SWE was performed by using a probe with an L9-4 linear array. Each patient was examined by two experienced radiologists (with $>3$ years of experience in musculoskeletal radiology) on the same day.

Both radiologists were blinded to each other's examination and to the physician's evaluations and to the patient's status.

\section{Patient positioning}

All US and SWE examinations were performed with the patient in the sitting position in front of an examination bed with their arms extended down by their side. The arms were extended and the forearms held in the supine position while the wrists rested on a flat surface. Care was taken to examine in a neutral position as much as possible. Patients were requested to remain as relaxed as possible throughout all testing. However, in some cases, spasticity and flexion posture may not allow holding the probe motionless in the correct position. In this case, a slight supination of the wrist was maintained on the paretic side to be able to insert the probe. The fingers were kept in a relaxed semiflexed position during the study. A standard US examination was initially performed to view the median nerve in the axial plane to delineate the anatomical structures better. Then images were obtained at the carpal tunnel level while the transducer was parallel to the long axis of the nerve (fig 1a). Longitudinal plane was preferred for SWE measurements to visualize the median nerve more widely from proximal carpal tunnel to distal carpal tunnel. If any movement occurred during SWE, the scanning was repeated. The radiologist kept the transducer motionless while performing SWE.

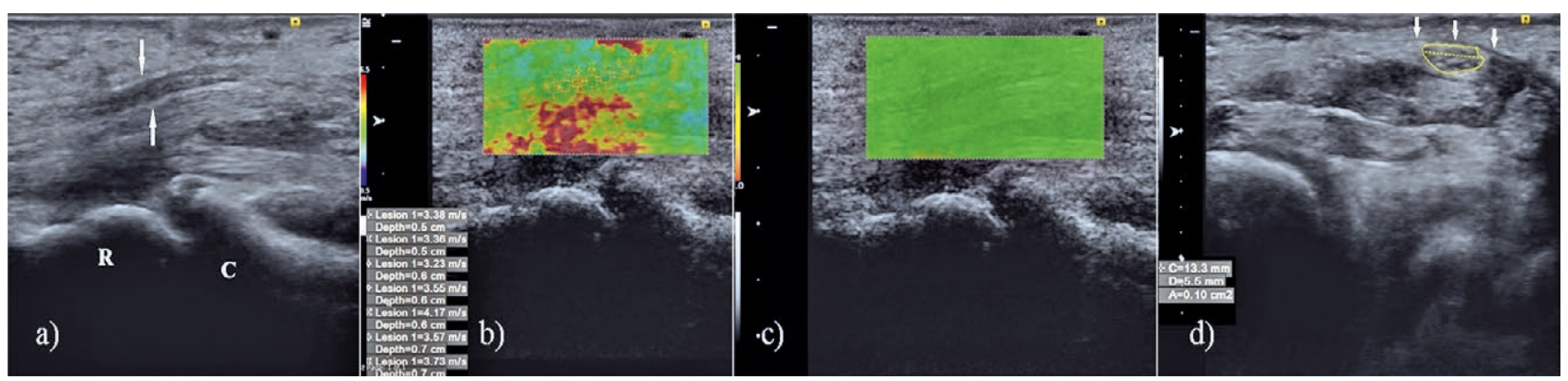

Fig 1. a) Sagittal B-mode US image of the median nerve (white arrows) at the carpal tunnel. $\mathrm{R}=$ distal radius, $\mathrm{C}=$ proximal carpal row; b) VTIQ map shows the measurement of stiffness at proximal and distal of the carpal tunnel; c) VTIQ quality control image, indicating high-quality shear waves; d) CSA measurement of the median nerve at the carpal tunnel inlet and the flexor retinaculum (arrows) overlying the median nerve were shown. 


\section{SWV measurements}

A rectangular electronic box-shaped region of interest (ROI) was used for SWV measurements automatically provided by the system software (fig 1b). Care was taken to place the ROIs at the median nerve. At least 5 separate SWE measurements were performed at each median nerve. Each patient's examination was repeated 3 times by the same physician on same day, and the mean value was used as the final result.

\section{Image quality assessment}

The quality of the images was assessed by color-coded quality maps provided by the US system in which the green areas were considered reliable (fig 1c). The yellow and red color-coded areas were considered as low-quality scans. If the image was of low quality, the scanning was repeated till high-quality images were obtained. All scans were repeated at least three times in each nerve, and the best representative image of the highest quality on the quality map was selected, which was used to measure SWV.

\section{CSA measurement}

A transverse imaging plane at the carpal tunnel inlet was obtained for measuring CSA of the median nerve (fig 1d). CSA measurements were done by performing a continuous boundary trace of the nerve.

\section{Statistical analysis}

Statistical analysis was performed using the statistical package SPSS software (version 17.0, SPSS Inc., IL, USA). If continuous variables were normal, they were described as mean \pm standard deviation $(P>0.05$ in Kolmogorov-Smirnov test or Shapira-Wilk $[n<30]$ ), and if they were not normal, they were described as the median. Comparisons between groups were applied using the Student $t$ test for normally distributed data and the Mann-Whitney $U$ test for data that were not normally distributed. The interobserver consistency test was applied with a variance analysis to determine the interclass correlation coefficient. An ICC greater than 0.80 indicated excellent agreement. Correlations between the duration of time since stroke, SWV and CSA of the median nerve were tested using the Pearson's correlation test. The correlation coefficients were interpreted as either excellent $r \geq 0.91$; good $0.90 \geq r \geq 0.71$; fair $0.70 \geq r \geq 0.51$; weak $0.50 \geq r \geq 0.31$; or little or none $r \leq 0.3$. Values of $p<0.05$ were considered statistically significant.

\section{Results}

Descriptive data (mean, standard deviation and frequency) on the demographic and clinical variables of the patient sample are presented in Table I.
Table I. Clinical characteristics of the study population

\begin{tabular}{ll}
\hline Characteristics & \\
\hline Age (years) & \\
Gender (females/ males),(n) & $56.5 \pm 11.56$ \\
Duration of stroke, months* & $10 / 14$ \\
Paretic side, right/left,( n) & $19.29 \pm 11.77$ \\
Stroke etiology, (n) & $10 / 15$ \\
$\quad$ Hypertension & 11 \\
$\quad$ Multifactorial & 8 \\
Diabetes Mellitus & 1 \\
Mitral valve surgery & 1 \\
Trauma & 1 \\
Unknown etiology & 2 \\
\hline
\end{tabular}

* mean \pm standard deviation, $\mathrm{n}$ - number

Table II. Mean cross-sectional area and SWV values of both readers for the affected and unaffected wrists

\begin{tabular}{llll}
\hline Parameter & Affected Side & Unaffected Side & $\mathbf{p}$ \\
\hline SWV $(\mathrm{m} / \mathrm{s})^{*}$ & $3.31 \pm 0.65$ & $3.08 \pm 0.51$ & $\mathrm{p}=0.03$ \\
$\mathrm{CSA}(\mathrm{mm} 2)^{*}$ & $6.84 \pm 1.21$ & $7.95 \pm 1.47$ & $\mathrm{p}<0.001$ \\
\hline
\end{tabular}

* Mean \pm standard deviation; SWV - Shear Wave Velocity

Table III. Interobserver variability for cross-sectional area and SWV measurements

\begin{tabular}{llll}
\hline Parameter & Reader 1* & Reader 2* & ICC (95\%) \\
\hline CSA, mm & & & \\
Affected side & $6.77 \pm 1.20$ & $6.91 \pm 1.25$ & $0.90(0.79-0.96)$ \\
Unaffected side & $7.96 \pm 1.44$ & $7.91 \pm 1.44$ & $0.94(0.88-0.98)$ \\
SWV, m/s & & & \\
Affected side & $3.32 \pm 0.64$ & $3.29 \pm 0.67$ & $0.91(0.81-0.96)$ \\
Unaffected side & $3.09 \pm 0.52$ & $3.07 \pm 0.521$ & $0.86(0.72-0.94)$ \\
\hline
\end{tabular}

CSA - cross-sectional area; * Mean \pm standard deviation; CI- confidence interval; ICC - interclass correlation coefficient

Mean CSA and SWV values of both readers for the affected and unaffected wrists were shown in table II. SWV of the median nerve at the affected side was significantly higher when compared to the unaffected side $(p=0.03)$ and CSA was significantly lower at the affected side when compared to the unaffected side $(p<0.001)$. There was a negatively fair correlation between the CSA of the median nerve at the affected side and duration of time since stroke $(r=-0.58)$. No significant correlation existed between the SWV of the median nerve at the affected side and the duration of time since stroke $(r<0.3)$. As shown in table III the interobserver agreement for CSA and SWV measurements were excellent.

\section{Discussions}

Upper limb spasticity may result in flexed wrist posture by involvement of the flexor muscles and this wrist posture has the potential to compress the median nerve at 
the carpal tunnel. This compression might also be related with the decreasing carpal tunnel area.

Our study demonstrated that the CSA of the median nerve on the affected side was significantly lower than that of the unaffected side and the median nerve stiffness increased on the affected side when compared to the unaffected side. Also, our study showed a negatively fair correlation between the CSA of the median nerve on the affected side and the duration of time since the stroke. It means that the CSA of the median nerve decreases as time passes by due to chronic compression. Histologically compression neuropathy causes edema, fibrosis, demyelination, and Wallerian degeneration [7]. The negative correlation among the CSA of the nerve and the duration of time since stroke means that the CSA of the median nerve decreases as the time of exposure to compression increases. This is probably related to the effects of chronic ischemia with secondary atrophy of the median nerve. Also, chronic compression may progress to fibrosis of the nerve in the chronic phase and our study demonstrated increase in the stiffness of the nerve when compared to the unaffected side. However uncorrelated results among the duration of time since stroke and the stiffness of the median nerve remain unclear.

Previously, CSA of the median nerve and stiffness of the median nerve was studied in carpal tunnel syndrome [8-10]. The deformity of the median nerve during wrist flexion or extension has already been shown and the CSA of the median nerve has been reported to decrease during wrist motion [5]. Also, the acute postural changes of the wrist have been reported to increase the median nerve stiffness [3]. Moreover, acute positional changes or acute increase in the pressure of the carpal tunnel may cause increase in both the intraneurial blood flow and the CSA of the median nerve due to edema [11]. Histologically chronic changes due to compression neuropathy are most prominent under the area of the directly compression. Our study demonstrated the atrophy of the median nerve and increased stiffness at the carpal tunnel due to chronic flexed wrist posture.

The first limitation of this study was the limited sample size. The second limitation was that the absence of peripheral neuropathy was not confirmed by performing electrophysiological studies on the unaffected side. Although the nonparetic extremities in patients with stroke are generally considered to be normal, ambulatory assistive devices, peripheral nerve conduction abnormalities or overuse of the nonparetic muscles may cause peripheral neuropathies of the median nerve on the unaffected side [12-14].

\section{Conclusions}

In conclusion, these results suggest that chronic flexed wrist posture may cause a decrease in the CSA of the median nerve due to chronic compression after stroke and increase in the stiffness of the median nerve. US and SWE may be helpful imaging modalities to detect the possible structural and anatomical changes due to chronic flexed wrist posture following stroke. Further larger and multi-center studies are needed to better define the effects of chronic flexed wrist posture on the elasticity and CSA of the median nerve among chronic stroke patients.

\section{Acknowledgments}

The authors would like to thank to Aysin Pourbagher for performing US and SWE as second radiologist.

\section{Conflicts of interest: None.}

Funding/Support: This study was supported and funded by the Institutional Research Fund.

\section{References}

1. van Wijck FM, Pandyan AD, Johnson GR, Barnes MP. Assessing motor deficits in neurological rehabilitation: Patterns of instrument usage. Neurorehabil Neural Repair 2006;15:23-30.

2. Bethoux F. Spasticity Management After Stroke. Phys Med Rehabil Clin N Am 2015;26:625-639.

3. Greening J, Dilley A. Posture-induced changes in peripheral nerve stiffness measured by ultrasound shear-wave elastography. Muscle Nerve 2017;55:213-222.

4. Loh PY, Muraki S. Effect of wrist deviation on median nerve cross-sectional area at proximal carpal tunnel level. Iran J Public Health 2014;43:180-185.

5. Loh PY, Muraki S. Effect of wrist angle on median nerve appearance at the proximal carpal tunnel. PLoS One 2015;10:e117930.

6. Wang Y, Zhao C, Passe SM, et al. Transverse ultrasound assessment of median nerve deformation and displacement in the human carpal tunnel during wrist movements. Ultrasound Med Biol 2014;40:53-61.

7. Thatte MR, Mansukhani KA. Compressive neuropathy in the upper limb. Indian J Plast Surg 2011;44:283-297.

8. Zhang C, Li M, Jiang J, et al. Diagnostic Value of Virtual Touch Tissue Imaging Quantification for Evaluating Median Nerve Stiffness in Carpal Tunnel Syndrome. J Ultrasound Med 2017;36:1783-1791.

9. Lin C, Loochtan AI, Dresser B, et al. Is carpal tunnel syndrome present in acute stroke patients? An investigative 
study using clinical and imaging screening tools. J Clin Neurosci 2017;39:111-113.

10. Kantarci F, Ustabasioglu FE, Delil S, et al. Median nerve stiffness measurement by shear wave elastography: a potential sonographic method in the diagnosis of carpal tunnel syndrome. Eur Radiol 2014;24:434-440.

11. Wilson KE, Tat J, Keir PJ. Effects of Wrist Posture and Fingertip Force on Median Nerve Blood Flow Velocity. Biomed Res Int 2017;2017:7156489.

12. Kellner WS, Felsenthal G, Anderson JM, Hilton EB, Mon- dell DL. Carpal tunnel syndrome in the nonparetic hands of hemiparetics. Stress-induced by ambulatory assistive devices. Orthop Rev 1986;15:608-611.

13. Sato Y, Kaji M, Tsuru T, Oizumi K. Carpal tunnel syndrome involving unaffected limbs of stroke patients. Stroke 1999;30:414-418.

14. Paoloni M, Volpe B, Mangone M, Ioppolo F, Santilli V. Peripheral nerve conduction abnormalities in nonparetic side of ischemic stroke patients. J Clin Neurophysiol 2010;27:48-51. 